\title{
Patrones de competencia y su efecto en los precios de los medicamentos de patente expirada en México: el caso de los antibióticos (2014-2015)
}

\author{
Eric Israel Rios Nequis \\ Lilia Domínguez Villalobos*
}

\begin{abstract}
RESUMEN
El mercado farmacéutico de patente expirada tiene un comportamiento interesante respecto a su estructura mercado, cambiando de una estructura monopólica derivada de la protección de patente a un oligopolio, donde la empresa que tenía dicha protección (pionera) ostenta el rol de dominante mediante en un juego a la Stackelberg, la cual aplica estrategias de diferenciación de producto en un mercado segmentado, el cual es poco sensible a los precios y que conserva una cuota no despreciable. En este sentido, otras empresas que fabrican genéricos con una marca comercial, penetran el mercado con una estrategia similar a la dominante, aunque con precios menores. Esto se debe al prestigio y posicionamiento competitivo del producto con reciente expiración. Consecuentemente, se ofertan productos poco diferenciados que compiten por precios económicos, los cuales obtienen una penetración de mercado considerable en un segmento sensible a los precios. Este comportamiento explica la variación de precios de medicamentos. Las estrategias que destacan son las dosificaciones, el prestigio del producto por medio de una marca y la línea de presentaciones, buscando un comportamiento de precios imperfecto y semejante a la paradoja de los genéricos, donde la política pública tiene capacidad para disminuir dichas fluctuaciones en la búsqueda de opciones económicas para la población.
\end{abstract}

Palabras clave: estructura de mercados, oligopolio, precios.

Clasificación JEL: L11, L13, L22.

* Doctor en Economía y profesor-investigador de la Universidad Autónoma de Baja California, Facultad de Ciencias Administrativas y miembro del Sistema Nacional de Investigación SNI Nivel C. email: eric. israel.rios.nequis@uabc.edu.mx. Autor corresponsal.

** Doctora en Economía, profesora-investigadora y miembro del Sistema Nacional de Investigación SNI Nivel III de la Facultad de Economía, Universidad Nacional Autónoma de México (UNAM). email: ldv@unam.mx 


\title{
Competition Patterns and their Effect on the Prices of Expired Patent Medicines in Mexico: the Case of Antibiotics
}

\begin{abstract}
The expired patent pharmaceutical market has an interesting behavior with respect to its market structure, changing from a monopolistic case derived from patent protection to an oligopoly, where the company that had such protection (pioneer) holds the dominant role through a Stackelberg behavior, which applies strategies of product differentiation in a segmented market, which is not very sensitive to change in prices and which retains a non-negligible share. In this sense, other companies that manufacture generics with a commercial brand, penetrate the market with a similar strategy with differentiated products and lower prices. This is due to the prestige and competitive positioning of the product with recent expiration. Consequently, little differentiated products are offered that compete for economic prices, which obtain a considerable market penetration in a price-sensitive segment. This behavior explains the variation in drug prices. The strategies that stand out are the dosages, the prestige of the product through a brand and the line of presentations, looking for imperfect price behavior and similar to the generic paradox, where public policy has the capacity to reduce such fluctuations in the search for economic options for the population.
\end{abstract}

Keywords: market structure, oligopoly, pricing.

JEL Classification: L11, L13, L22.

\section{INTRODUCCIÓN}

Schumpeter (1965) planteó que el monopolio es necesario para que las empresas realicen investigación y desarrollo que puede resultar en productos que satisfagan mejor las necesidades o que tengan menores costos de producción. Sin duda, sin el aliciente de las ganancias estas empresas no tendrían ningún incentivo para enfrascarse en esta actividad. Por tanto, las patentes son uno de los pilares de la innovación para la industria farmacéutica, dado que el costo para realizar ingeniería a la inversa en esta industria es marginal (Kim, 1997). Las nuevas medicinas deben recuperar los costos de investigación desarrollo antes de obtener un beneficio o rentabilidad, las cuales, son razones suficientes para fijar precios elevados en sus productos innovadores. La ventaja de las patentes tiene una terminación legal de duración en todos los países, que va de doce a veinte años con el fin de limitar el poder de monopolio de las empresas. Así, cuando las patentes expiran, los precios de los medicamentos deben descender ante la amenaza de entrada de nuevos laboratorios: los escenarios clásicos enfatizan una reducción de precios simultánea por el ingreso de ofertantes con productos a precios módicos.

Empero, los precios de los medicamentos de patente expirada no se comportan competitivamente como prediría la teoría microeconómica tradicional. Scherer (1993) 
señala que los precios de los medicamentos pioneros (de reciente expiración), no disminuyen cuando ingresa la competencia de genéricos y se refiere a este comportamiento de precios como la paradoja de los genéricos. Hay hallazgos en la evidencia empírica de autores asociados a un comportamiento precios constantes o rígidos e incluso hay hallazgos que indican aumentos ante la entrada de competencia (Frank y Salkever, 1997; Suh, Manning, Schondelmeyer y Hadsall, 2000; Saha, Grabowsky, Birnbaum, Greenberg y Bizan, 2006; Reagan, 2008; Vandoros y Kanavos, 2013), algunos trabajos modelan teóricamente este comportamiento (Frank y Salkever, 1992 y Papanastasiou, 2016). El hecho es que el mercado de medicamentos de patente expirada es altamente segmentado y se pueden reconocer tres grupos de medicamentos: aquellos que originalmente estaban protegidos por la patente y que tienen una marca acreditada (medicamentos pioneros), medicamentos que entran a competir con marca (genéricos con marca) y presentaciones específicas y los que se reconocen con la sustancia genérica y no tienen una presentación que los diferencie ni marca (Wiggins y Maness, 2004; Danzon y Furukawa, 2003 y 2008).

El objetivo de este trabajo es determinar los factores que contribuyen a explicar las variaciones de precios en el mercado de patente expirada. En particular, se analiza la posición competitiva que tienen los distintos jugadores y sus asociaciones en los precios. El artículo pretende demostrar que la competencia perfecta no rige el mercado de patente expirada en México y que este escenario sugiere la presencia de modelos de empresa dominante o pares que suponen oligopolios diferenciados y la presencia de barreras de entrada. Lo anterior plantea la necesidad de regular los mercados en lugar de suponer que las fuerzas libres de la competencia harán bajar o declinar los precios.

Bajo un contexto donde hay entrada de empresas competidoras, se busca probar que las compañías farmacéuticas pioneras (patentadoras), defienden su mercado al expirar la patente con acreditación de marca, innovaciones comerciales en las dosificaciones por día y adiciones nuevas en la vía de administración (supositorios, cápsulas, jarabes, suspensiones trihidratadas, etc.), lo que se traduce en precios mayores a los medicamentos de los otros segmentos. El resultado es una estructura de carácter oligopólico y diferenciado en la que participan los tres tipos de medicamentos mencionados en líneas anteriores y la competencia en este mercado distinta a los precios es predominante, explicando con ello la dispersión de precios entre los distintos productos (paradoja de los genéricos).

El trabajo consta de tres secciones adicionales a esta introducción. Primero se revisan los principales trabajos en la literatura que aborda el comportamiento de las empresas en este mercado con el fin de establecer hipótesis de comportamiento de los precios en el mercado de patente expirada. En seguida, se examina la estructura farmacéutica mexicana en los diversos eslabones de la cadena valor y el análisis de precios con metodología cuantitativa de los antibióticos con patente expirada en México. Finalmente se presentan conclusiones y recomendaciones de política pública. 


\section{MARCO TEORICO DE REFERENCIA}

A la paradoja de los genéricos (Scherer, 1993), se le atribuyen diversos elementos de comportamiento estratégico en la organización industrial y de política pública como agentes explicativos a dicho comportamiento de precios.

La industria farmacéutica tiene una estructura oligopólica donde se ofrecen productos heterogéneos con un componente químico homogéneo. Al expirar la patente, se establece un productor que tiene una participación grande en el mercado y otros de menor tamaño que siguen el comportamiento de este (Schettino, 2003). Según el autor, la forma de competencia es de empresa dominante o líder-seguidor, porque la compañía líder busca maximizar sus ganancias, actuando de manera similar a un monopolio, mientras que los seguidores tratan de que no los desplace del mercado, actuando de manera similar a la competencia perfecta. Por tanto, en este comportamiento el líder debe realizar esfuerzos para mantener dominancia y los seguidores deben esperar a que los costos de la empresa dominante aumenten para intercambiar posiciones.

Los precios fungen como indicativos de altas ganancias y de poder monopólico. Por el contrario, si los precios disminuyen, indican mayor rivalidad en la industria y ganancias en declive. Es así que se plantea la paradoja de los genéricos porque no cumple los preceptos microeconómicos tradicionales y es discutida por los distintos analistas. Por un lado existe evidencia que argumenta un leve descenso de los precios de los medicamentos pioneros para mercados como el estadounidense. Según la evidencia, los precios pueden declinar un $2 \%$ por cada competidor de genéricos que ingrese o un $22 \%$ con 20 competidores para un medicamento o componente químico (Caves, Whinston y Hurwitz, 1991). Otros estudios más actualizados relacionan un descenso de $0,2 \%$ por cada entrante (Saha et al., 2006). Y en términos monetarios para el caso de los antibióticos en Estados Unidos, se encuentra que el precio de los medicamentos pioneros puede disminuir 60 dólares el entrar un competidor, 30 cuando entran dos o tres competidores, 20 cuando hay cuatro competidores y se establece una relación de asociación donde al entrar un competidor, el precio puede disminuir cuatro dólares en contextos con 40 competidores o más (Wiggins y Maness, 2004). Por el contrario, hay evidencia que indica aumentos en los precios de los medicamentos pioneros ante el ingreso de competidores en mercados donde no hay regulación económica (Grabowsky y Vernon 1992, Frank y Salkever, 1997 y Reagan, 2008) y casos donde hay regulación, como los mercados europeos (Magazzini Pammoli y Ricabonni, 2004; Vandoros, y Kanavos, 2013) y donde su efecto puede ser variable acorde a la clase terapéutica y la cantidad de competidores (Mueller y Frenzel, 2015), es decir hay debate en torno a la respuesta que pueda originar las libres fuerzas del mercado en los productos farmacéuticos que ya no son protegidos por una patente.

Detrás de este comportamiento de precios han sido señaladas las acciones estratégicas, de las empresas para defender su nicho de mercado (Frank y Salkkever, 1992) 
y evitar la declinación de precios (Frank y Salkever). Esta segmentación también se suscita en los mercados regulados (Kanavos, Costa-Font y Seeley, 2008). Hay evidencia importante sobre preservación de altas cuotas de mercado por parte de los fabricantes de medicamentos pioneros u originales en valores, respecto al volumen (Suh et al., 2000; Danzon y Furukawa, 2003 y 2008)). Mientras las empresas de genéricos buscan un desarrollo de mercado por precios atractivos, mismos que declinan conforme transcurre el tiempo (Caves, Whinston y Hurwitz, 1991; Saha et al., 2006). Esta dinámica no genera presión suficiente para reducir precios porque emplean estrategias para disuadir la entrada y diferenciarse de los rivales.

En un escenario de competencia ante la expiración de patente, se ubican tres competidores en el mercado farmacéutico. La empresa pionera que enfrenta la expiración de patente, la cual tiene tiempo comercializando su fármaco durante el tiempo de protección de patente. Seguido de medicamentos con marca respaldada por empresas farmacéuticas de tamańos y capacidades equiparables a la empresa líder (patente expirada) y medicamentos económicos sin marca que se comercializan con el componente químico y el sello de genéricos intercambiables, los cuales no tienen presentaciones e innovaciones comerciales en demasía como los dos primeros competidores mencionados.

Las acciones para defender el nicho por parte de las empresas pioneras, según la literatura son de carácter estratégico para disuadir la entrada. La diferenciación es el elemento principal de estudio y se aborda desde un enfoque de producto, el cual es evaluado por la marca y el desarrollo de producto. Las más importantes son:

a) Ventaja de ser el primero y economías de escala (Frank y Salkever, 1992 y Ferrándiz, 1999; Kong y Seldon, 2004; Kamien y Zang, 2009; Granier y Trinquard, 2010 y 2012).

b) Diferenciación y lealtad a la marca (Hurwitz y Caves, 1988; Caves, Whinston y Hurwitz, 1991; Grabowsky y Vernon, 1992).

c) Segmentación de mercados e introducción de versiones genéricas de los laboratorios de patente (Frank y Salkever, 1997; Hollis, 2002 y 2005; Reiffen y Ward, 2007; Ferrara y Kong, 2008; Reagan, 2008; Granier y Trinquard, 2010 y 2012; Rodrigues, Gonçalves y Vasconcelos, 2014).

d) Modificaciones de producto (Hong, Shepherd, Scoones y Wan, 2005; Bhat, 2005; Pearce, 2006; Clift, 2008), tales como mejoras en las presentaciones, que tienen como objetivo reducir la frecuencia de aplicación en un tratamiento; adicionalmente, los productos innovan en la vía de administración para ofrecer una mayor variedad de opciones a los médicos en la prescripción de un medicamento, surgen presentaciones en pomadas, suspensiones tri-hidratadas, tabletas dispersables o de absorción más rápida, sublinguales, etc. 
Por otro lado, es importante considerar elementos que inducen la competencia. Uno de ellos es la política pública. La alta participación de los sistemas públicos en el sector salud o alta cobertura en los seguros médicos, teóricamente buscarán productos económicos para contener sus gastos farmacéuticos, bajo el supuesto del ahorro en el caso institucional y la cobertura como limitante en el sector privado (Scott, 1999 y 2000; Iizuka, 2009).

\section{UN MODELO TEÓRICO DE EMPRESA FARMACÉUTICA PIONERA}

Dentro de la teoría económica de la competencia imperfecta, se ofrece un modelo que tiene como característica fundamental que una de las empresas se adelanta a las otras y que a través de esta acción y solo esta, logra obtener una mayor ganancia. Este modelo fue originalmente propuesto por Stackelberg en 1934 (von Stackelberg, 1952) y puede ilustrar el comportamiento de las empresas pioneras. Originalmente la empresa puede ser, en situación de duopolio, incluso idéntica a la otra pero elige sus cantidades primero que la otra, es decir las cantidades se eligen en forma secuencial y no simultanea como en Cournot. Sin embargo desarrollos posteriores a la teoría aportan mayores elementos para este tipo de modelos en que hay una amenaza de entrada. Esto se refiere a la creación de asimetrías que hagan creíble la fortaleza de la empresa incumbente (Tirole, 1988), que para el análisis en este artículo, es la empresa pionera (Frank y Salkever, 1997). En este contexto, los costos de las empresas son desiguales, pero también se presentan distintas elasticidades en la demanda en cada una; y la más inelástica es la de la empresa pionera por lo que podrá tener un premio extra sobre su costo debido a la lealtad de su mercado. Para la industria farmacéutica varios autores entre los que destacan Frank y Salkever (1992 y 1997), Kong (2004), Reagan (2008) y Kanavos, Costa-Font, y Seeley (2008) han modelado este comportamiento.

Este último ilustra de manera satisfactoria nuestro planteamiento:

Sea la función de demanda

(1)

$$
P_{i}=P(Q)
$$

en donde

(2)

$$
Q=q_{i t}+N_{-i t}
$$

El subíndice i se refiere al precio que fija la empresa pionera y -i a los competidores de genéricos. De la cantidad de $\mathrm{N}$ competidores de genéricos, asumimos que $\mathrm{N}-1$ son seguidores Stackelberg y sustitutos estratégicos $\left(\frac{\partial \mathrm{q}_{\mathrm{i}}}{\partial \mathrm{q}_{-\mathrm{i}}}<0\right)$. Suponiendo una función de ganancias dos veces diferenciable y cóncava. Por tanto, hay una función de reacción $q_{i}=R_{i}\left(\sum q_{-i}\right)$, es así que la función de ganancia es:

$$
\Pi^{i,-i}=q_{i} P_{i}\left(q_{-i}+R\left(\sum q_{-i}\right)\right)-K
$$


Donde $\mathrm{K}$ se refiere a los costos sumergidos, dado que las compañías de genéricos afirman ser igualmente competentes en cantidad $\sum q_{-i}=q\left(N_{t}\right)$, la función de reacción de la empresa pionera es representada por:

$$
q_{i}=R_{i}\left(q_{i t}\right)=q\left(P_{i t}, N_{t}\right)
$$

Como resultado, el precio de equilibrio para el medicamento pionero será determinado por la siguiente función:

$$
Q=q_{i}+q\left(N_{t}\right)
$$

y resulta ser:

(6)

$$
P_{i t}=A\left(q_{i}, N_{i t}\right)
$$

La ecuación 6 sugiere que el precio del fármaco pionero continuará declinando a causa del aumento constante de competidores de genéricos en el mercado. Estos resultados pueden verse afectados por la colusión entre los competidores reales, y los potenciales podrían determinar una forma de función de reacción no enfocada a reducir precios, porque asumen que el precio es determinado por la regulación más que por la competencia, es así que los nuevos entrantes pueden decidir competir por los atributos de productos o por ofrecer mejores condiciones a los distribuidores, como mejoras en los acuerdos comerciales. En este sentido, el patrón de diferenciación en el mercado de genéricos, pareciera desarrollarse como una diferenciación de producto horizontal (Frank y Salkever, 1997), y está basada en las características del producto, como las formas de dosificación por molécula, vías de administración (tableta, supositorio, cápsula, píldora, inyección, etc.), características del empaque y etiquetas, más que en una diferenciación vertical donde los atributos de la calidad del producto son determinantes para generar lealtad a la marca.

Bajo este supuesto, la penetración de genéricos y su precio son determinadas por las compañías para obtener la ganancia deseada, después de enfrentar la entrada (costos sumergidos), ajustando su producto a las características requeridas de país en país con el fin de diferenciar su producto. Mientras, la firma pionera no necesariamente se verá obligada a reducir sus precios. Por tanto, el modelo de penetración de genéricos, se representaría así:

$$
q_{-i}=f\left(N, P_{i}, \omega_{1}, R, W, R\right)
$$

Donde la penetración de genéricos $\left(q_{-i}\right)$ depende de la estructura del mercado $(\mathrm{N})$, los precios actuales de cada producto $\left(P_{i}\right)$, el cual afecta potencialmente el margen de precio, una vez que ingresa un producto al mercado junto con sus características propias, las cuales afectan la cantidad $\left(\omega_{1}\right)$, el reembolso o sistema de copago $(R)$, las regulaciones en el sector de distribución mayorista $(W)$ y las acciones en el eslabón detallista $(R)$. Estos elementos afectarán directamente la penetración de los genéricos. 
(8)

$$
P_{i}=f\left(N, q_{i}, \omega_{2}, R, W, R\right)
$$

Los precios serán determinados en base a la penetración de genéricos $\left(q_{-i}\right)$, la cual afecta las ganancias o beneficios, otro factor es la estructura del mercado $(N)$, las características de los productos $\left(\omega_{2}\right)$, la política pública del sistema de reembolsos $(R)$. Los autores señalan que regulaciones en los eslabones de distribución solo afectarán los alcances de la penetración.

El trabajo teórico de los autores considera diversos elementos para explicar las variaciones de los precios en el mercado fuera de patente. Sin embargo, la bibliografía todavía no determina detalladamente el comportamiento de la competencia, ya que no discrimina a las empresas seguidoras por el tipo de producto que comercializan (marca/sin marca). Esto permite deducir que se pueden construir las barreras a la entrada, como la diferenciación (Bain, 1956), la cual se traduce en las características de los productos dentro de su modelo. Estos factores ayudan a establecer una propuesta empírica para explicar las variaciones de precios entre los distintos productos ofertados en el mercado mexicano donde la patente ya expiró. El modelo empírico será analizado, discutido y demostrado en la sección de metodología y resultados.

La expresión es la siguiente:

$$
P=f\left(P_{o}, G_{M}, G_{I}, D_{p}, E_{M}, P_{P}, C_{I}\right)
$$

Donde $\left(P_{o}\right)$ es el precio del medicamento pionero o de reciente expiración, $\left(G_{M}\right)$ el genérico con marca o producto seguidor que competirá con una marca y estrategia similar al medicamento de reciente expiración, $\left(G_{I}\right)$ el genérico sin marca o intercambiable que compite por precio y no posee una marca comercial, $\left(D_{p}\right)$ diferenciación de producto mediante presentaciones y dosificaciones, $\left(E_{M}\right)$ la estructura de mercado, $\left(P_{p}\right)$ política pública o cuadro básico de medicamentos (presentaciones de medicamentos que son económicos y eficientes, según la secretaria de salud y $\left(C_{I}\right)$ comercio internacional.

\section{ESTRUCTURA DE MERCADO Y EVOLUCIÓN DE LOS MEDICAMENTOS GENÉRICOS EN MEXICO}

La estructura de mercado de la industria farmacéutica en el mercado de los genéricos es fundamental para su análisis porque nos permite conocer la complejidad de esta y entender el desarrollo que estos productos han logrado en la actualidad. 


\section{Gráfico 1. Participación porcentual de los distintos medicamentos en México y su relación valores/volumen (2012) \\ -Volumen Valores Relación val/vol}

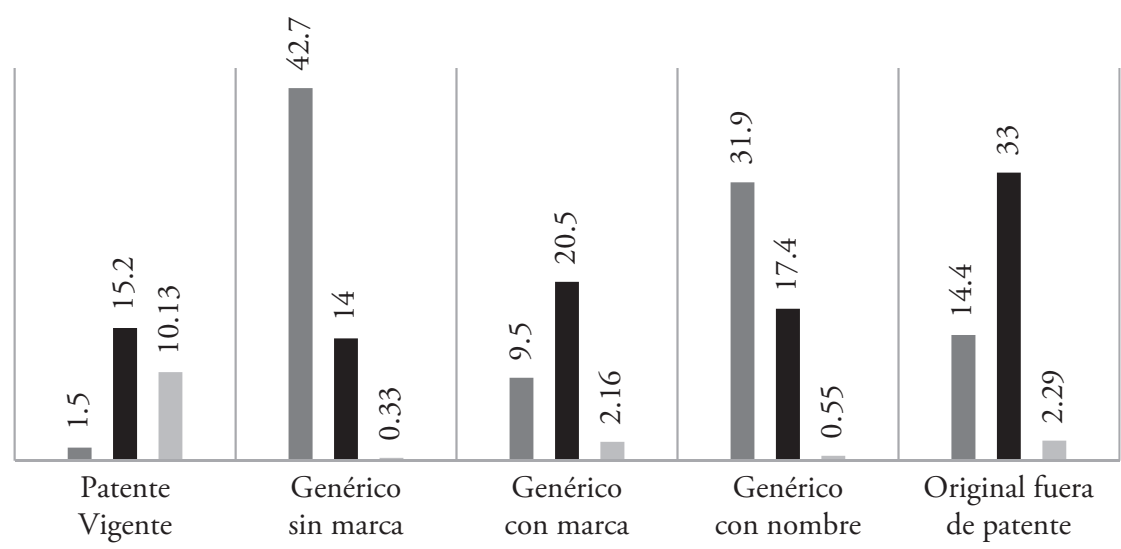

Fuente: Funsalud con datos de IMS Health (2013).

El mercado farmacéutico mexicano obtuvo ventas por 114 mil millones de pesos en 2009, según datos de la Encuesta Industrial Anual, de los que 4 millones corresponde al subsector dedicado a la elaboración de materias primas, y los 110 millones restantes corresponde al homólogo enfocado a fabricar preparaciones farmacéuticas.

En 2012, según datos de Funsalud (2013), el mercado estuvo valuado en 199 mil millones de pesos, donde los fármacos con patente expirada obtuvieron el 98,5\% de las ventas en volumen y el $84,8 \%$ en valores (168 mil millones setecientos cincuenta y dos mil pesos). Dentro de ese mercado, figuran los medicamentos genéricos sin marca, los cuales obtuvieron una cuota de mercado 14 por ciento en valores $(23$ mil millones seiscientos veinticinco mil doscientos ochenta pesos) y 42,7 en volumen, seguido de los genéricos con marca, que registraron el $20,5 \%$ de la ventas en valores ( 43 mil millones quinientos noventa y cuatro mil y ciento sesenta pesos) y $9,5 \%$ en volumen.

En tercer lugar están los genéricos con nombre, quienes abarcaron el 17,4 por ciento de las ventas en términos monetarios $(29$ mil millones trescientos sesenta y dos mil ochocientos cuarenta y ocho pesos), y el 31,9 por ciento en volumen. Finalmente están los medicamentos originales fuera de patente (pioneros), los cuales alcanzaron una participación de 33 ( 55 mil millones seiscientos ochenta y ocho mil ciento sesenta pesos) y 14,4 por ciento en valores y volumen respectivamente (gráfico 1).

El gráfico 1 indica que la relación ventas en valores/volumen, las marcas pioneras son las que mayor coeficiente tienen es decir tienen una relación de 2,29, seguido de los genéricos con marca $(2,16)$, los genéricos con nombre $(0,55)$ y los genéricos económicos o sin marca $(0,33)$, lo que indica una competencia distinta a los precios. 
La información se confirma con datos de IMS Health para el mismo período donde del 100 por ciento de las ventas en valores y volumen para los períodos 2011 y 2012 respectivamente. Bajo un enfoque de segmentación de mercados las marcas originales tienen un desempeño importante en el mercado privado y público tanto en valores, como en volumen porque su coeficiente de relación valores volumen es mínimo de 1.80 para el caso institucional y de 2,67 para el privado. Por su parte, los genéricos tienen lo propio con una relación de 2,11 para el mercado privado únicamente. Mientras que los genéricos poco diferenciados o sin marca de .55 y . 17 para el mercado privado e institucional respectivamente, lo que demuestra un comportamiento de dispersión de precios para ambos segmentos (cuadro 1).

Cuadro 1. Penetración de mercado de los medicamentos fuera de patente en sus distintos segmentos en México (2012)

\begin{tabular}{lcccccc}
\hline & \multicolumn{2}{c}{ Valores } & \multicolumn{2}{c}{ Volumen } & \multicolumn{2}{c}{ Valores/Volumen } \\
\hline $\mathbf{2 0 1 1}$ & Privado \% & Público \% & Privado \% & Público \% & Privado & Público \\
\hline Marcas originales & 41 & 19 & 17 & 10 & 2,41 & 1,90 \\
Genéricos sin marca & 17 & 5 & 29 & 35 & 0,59 & 0,14 \\
Genéricos con marca & 19 & & 9 & & 2,11 & 0 \\
Total & 100 & 14629 & 100 & 3049 & & \\
\hline 2012 & Privado \% & Público \% & Privado \% & Público \% & & \\
\hline Marcas originales & 40 & 18 & 15 & 10 & 2,67 & 1,80 \\
Genéricos sin marca & 17 & 6 & 31 & 36 & 0,55 & 0,17 \\
Genéricos con marca & 19 & 0 & 9 & 0 & 2,11 & 0 \\
Total & 100 & 15309 & 100 & 3151 & & \\
\hline
\end{tabular}

Fuente: Elaboración propia con información de salud y fármacos (2013).

Los laboratorios que comercializan medicamentos de patente expirada pueden ser fabricados directamente o buscar otra empresa que lo manufacture y ellos comercializarlo. Por otro lado, pueden aplicar las dos estrategias por cuestiones de capacidades productivas y manejo eficiente de costos de fabricación.

En México hay 18 empresas de genéricos (10 de capital nacional y 8 de extranjero) que fabrican sus propios medicamentos, las cuales son de tamańo mediano y que segmentan su mercado para comercializar medicamentos al sector institucional mediante licitaciones e introducen genéricos con marca comercial en el sector privado, donde se compite por promoción de ventas en el consultorio médico o en las farmacias (anaqueles y merchandising) en el caso de los libres de prescripción (OTC).

Por otro lado, se ubican ocho compañías de tamaño mediano, donde dos figuran dentro de las más importantes por sus ventas, las mencionadas comercializan medicamentos de manufactura propia y de tienen siete grandes fabricantes de capital nacional 
que les abastece lo que no producen, estas empresas, normalmente incursionan en el mercado farmacéutico privado, debido a las ganancias que este otorga, que como se verá más adelante en el artículo, es el nicho donde los genéricos con marca se desarrollan.

En ciertos casos, algunas maquiladoras forman parte de consorcios de farmacéuticas grandes. Finalmente, algunas empresas ofertan medicamentos de manufactura externa $\mathrm{u}$ otras que cuentan con laboratorios como principal proveedor y manufacturero. Los medicamentos genéricos intercambiables han logrado un desarrollo de mercado importante porque crecieron $32,23 \%$ por año en el período $2007-2009$, es decir, su producción aumentó de 25,8 millones de unidades a 45,1 millones, según datos de la consultora Knobloch (2009).

La Asociación Mexicana de Fabricantes de Genéricos Intercambiables (AMEGI) reporta 2903 medicamentos genéricos, los cuales se distribuyen en diferentes clases terapéuticas para el año 2009. Las áreas más representativas son los antibióticos, anti-infectivos, oncológicos, gastroenterológicos, endocrinológicos, analgésicos, cardiológicos, dermatológicos, neumológicos y reumatológicos, que en conjunto representan el 78,8\% del total de los medicamentos genéricos intercambiables (AMEGI, 2010).

Según el organismo citado anteriormente, la mayor parte de estos medicamentos (90\%) están registrados en el cuadro básico de medicamentos de la Secretaría de Salud, es decir, 470 de los 522 que conforman dicho cuadro, el cual debe ser tomado como referencia por las diferentes instituciones públicas de salud. En el cuadro básico de medicamentos, los antibióticos son la principal clase terapéutica en México, porque se conforma de 90 medicamentos, es decir, el 17,2\% del total de los considerados esenciales; le siguen vacunas; medicamentos cardiológicos, gastroenterológicos, hematológicos y neurológicos. En resumen, los medicamentos genéricos buscan desarrollarse en clases terapéuticas crónicas y degenerativas.

Analizar la cuota de mercado de las principales empresas que fabrican o comercializan genéricos es importante porque nos permite apreciar los distintos crecimientos en valores y volumen. En párrafos anteriores, se ilustró las variaciones en la cuota de mercado de los distintos productos. En estas líneas, se plasmarán las asimetrías por sus ventas de los principales jugadores en la industria de genéricos.

Datos de Knobloch (2008) permiten observar que entre las 15 empresas más importantes por sus ventas, 11 empresas que tienen medicamentos de patente tienen mayor crecimiento por el monto de sus ventas que las registradas en volumen, esta asimetría indica que los productos genéricos poseen precios elevados y plurales entre los distintos ofertantes, los cuales son de capital nacional y extranjero. Las empresas en cuestión, tienen la capacidad de comercializar medicamentos (genéricos con marca y sin marca) en el mercado privado e institucional. Por el contrario, se ubican empresas cuatro empresas que tiene un crecimiento mayor en volumen que en valores, lo que explica precios menores en sus productos, respecto a las otras once empresas. 
Por otro lado, el crecimiento medio anual de 2003 a 2007 de las empresas nacionales (13,52 y 11,47 por ciento en valores y volumen) fue mayor que sus homólogas extranjeras (5,98 y 12,82 por ciento). Las empresas de genéricos desde un enfoque generalizado obtuvieron crecimientos de 12,82 y 8,71 por ciento en valores y volumen, lo que confirma la dispersión de precios. Una de las explicaciones al fenómeno es la segmentación de mercados es confirmada en Frank y Salkever (1992) y Grabowsky y Vernon (1992), donde teorizan que las empresas farmacéuticas aprovechan ese cambio en la estructura de mercado para ofrecer productos acorde a las distintas elasticidades y preferencias de los distintos consumidores.

Esto sugiere que al menos en este tipo de empresas seguidoras no se está dando un declive de precios, como sería de esperarse en un mercado donde la patente ya expiró y se suscita la entrada de competidores.

El cuadro 2 indica la posible presencia de la paradoja de los genéricos en México donde los competidores de genéricos tienen una erosión de precios tal y como lo establece la literatura. Mientras, los medicamentos pioneros u originales con patente vencida preservan su precio o incluso aumentan levemente (Grabowsky y Vernon, 1992; Frank y Salkever, 1997; Suh et al., 2000; Wiggins y Maness, 2004; Reagan, 2008).

Cuadro 2. Nivel de precios correspondiente los meses de entrada de competidores en México de 2009 a 2015

\begin{tabular}{lccc}
\hline \multicolumn{1}{c}{$\begin{array}{c}\text { Precio promedio relativo correspondiente } \\
\text { a medicamentos }\end{array}$} & \multicolumn{3}{c}{$\begin{array}{c}\text { Meses posteriores a la entrada } \\
\text { del primer genérico }\end{array}$} \\
\hline & 6 & 12 & 24 \\
Genéricos (distinto al medicamento original) & $-20,60 \%$ & $-23,20 \%$ & $-28,60 \%$ \\
Originales (de patente vencida) & $2,50 \%$ & $1,30 \%$ & $0,40 \%$ \\
\hline
\end{tabular}

Fuente: COFECE, 2017.

Por otro lado, el cuadro 3 muestra que la penetración de competencia de genéricos es lenta en el mercado privado. Los datos señalan que a dos años del primer entrante de genéricos, estos productos ocupan el 24 por ciento de las ventas en volumen para el segmento de mercado citado.

Cuadro 3. Penetración de los genéricos 6, 12 y 24 meses de su lanzamiento (2009-2015)

\begin{tabular}{llcc}
\hline $\begin{array}{l}\text { Participación promedio en volumen de los } \\
\text { distintos genéricos respecto al medicamento } \\
\text { original en el mercado privado }\end{array}$ & \multicolumn{3}{c}{$\begin{array}{c}\text { Meses posteriores a la entrada } \\
\text { del primer genérico }\end{array}$} \\
\cline { 2 - 5 } & $6 \%$ & $12 \%$ & $24 \%$ \\
\hline
\end{tabular}

Fuente: COFECE, 2017.

La información presentada sugiere un escenario parecido al presentado en la literatura estadounidense donde la empresa pionera pareciera aprovechar su posición dominante 
en la industria, aprovechando sus ventajas en capacidades y tamaño para preservar su liderazgo en precios y conservar una proporción importante de mercado muy rentable donde no hay sensibilidad alta a los precios y se puede generar lealtad a la marca (el segmento privado). En este tenor una porción de seguidores compiten con una estrategia diferente a precios económicos en el mismo segmento mencionado, fomentando con ello variabilidad de precios en los productos ofertados. En la siguiente sección se hablan de los obstáculos que tienen los medicamentos genéricos.

\section{BARRERAS A LA ENTRADA QUE ENFRENTAN LOS MEDICAMENTOS DE GENÉRICOS}

La entrada a la World Intellectual Property Organization (WIPO) extendió la duración de las patentes y por tanto dificultó la entrada de los medicamentos de patente expirada hasta que estas se vencieran. El cuadro 4 muestra la lenta penetración de los genéricos intercambiables en el mercado farmacéutico mexicano como resultado en parte de las barreras legales, los cambios en la estructura productiva y los obstáculos originados por la distribución mayorista y detallista en razón de su poder de negociación.

Lo anterior contrasta con mercados como el estadounidense, canadiense, francés y alemán, donde a dos años de la entrada del primer competidor de genéricos la cuota de mercado de los genéricos sin marca en el primer país era de 70\% para 1998-2001 y pasó a $92 \%$ para 2006-2009. Las cuotas en los otros países fueron 31\% y 67\% en Reino Unido, de $62 \%$ y $54 \%$ en Canadá y de $16 \%$ y $57 \%$ en Francia. En México la participación de mercado de los productos mencionados era inexistente a dos ańos de la entrada del primer genérico de 1998 a 2001, y de 1\% de 2006 a 2009. Mientras, la penetración de los genéricos o seguidores con marca era de $43 \%$ en el período 1998-2001, y de $29 \%$ de 2006 a 2009 (cuadro 4).

Cuadro 4. Cuota de mercado de los medicamentos genéricos en México a dos años de la entrada del primer competidor de genéricos (1998-2001, 2006-2009)

\begin{tabular}{lcccc}
\hline \multirow{2}{*}{\multicolumn{1}{c}{ País }} & \multicolumn{2}{c}{ Genéricos sin marca $\%$} & \multicolumn{2}{c}{ Genéricos con marca \% } \\
\cline { 2 - 5 } & $\mathbf{1 9 9 8 - 2 0 0 1}$ & $\mathbf{2 0 0 6 - 2 0 0 9}$ & $\mathbf{1 9 9 8 - 2 0 0 1}$ & $\mathbf{2 0 0 6 - 2 0 0 9}$ \\
\hline E.U.A & 70 & 92 & 4 & 1 \\
Reino Unido & 31 & 67 & 16 & 0 \\
Canadá & 62 & 54 & 7 & 22 \\
Francia & 16 & 57 & 1 & 4 \\
Alemania & 32 & 50 & 25 & 15 \\
Italia & 3 & 17 & 58 & 15 \\
Espańa & 9 & 17 & 17 & 3 \\
Japón & 3 & 8 & 8 & 5 \\
Brasil & 2 & 41 & 43 & 15 \\
México & 0 & 1 & 43 & 29 \\
\hline
\end{tabular}

Fuente: Danzon y Furukawa (2011) con datos de IMS Health. 
Resulta claro que con el modelo de propiedad intelectual se aprecian varios actores e intereses. Por una parte, están los intereses de los países y los de las empresas que centralizan la innovación y por otra los de las empresas de genéricos que intentan aprovechar los nichos que se han generado por la expiración de las patentes, lo que da lugar a múltiples estrategias para defender posiciones (Mizrahi, 2010).

Guzmán y Pluvia (2004) señalan que en el actual régimen de competencia las farmacéuticas extranjeras tienen mayor interés en explotar sus productos farmacéuticos novedosos en nuevos mercados debido a la protección intelectual, lo cual debe reflejarse en un mayor número de nuevos tratamientos terapéuticos, mayor abasto de medicamentos y en consecuencia mejores servicios de salud. Sin embargo, señalan que no toda patente implica de modo necesario una nueva molécula, debido a la estrategia que aplican las empresas de identificar nuevos usos terapéuticos para prolongar la vida de la patente.

Por otro lado, las autoras comentan que hay dos hechos que se deben considerar para deducir que no necesariamente los medicamentos que ingresaron a partir de la reforma de la propiedad intelectual son los más avanzados. En primer lugar, que aumentó considerablemente la concesión de patentes de 1991 a 2002. En segundo, se incrementó la importación de medicamentos.

A continuación presentamos las principales barreras a la entrada que enfrentan en el mercado farmacéutico mexicano:

\section{a) Barreras legales}

La primera barrera legal enfrentada por los productores de genéricos sucede con la entrada de México a la Organización Mundial de Propiedad Intelectual (OMPI) y la extensión de las patentes a veinte años en 1993. Esto retrasó su entrada hasta que expiraran las mencionadas.

En materia de propiedad intelectual, el sector farmacéutico experimentó un auge de solicitudes de patente, provenientes de empresas transnacionales. En el período de 1980-1990 se otorgan 930 a la industria. Una década después (1991-2002) el patentamiento se triplicó (cuadro 5).

Cuadro 5. Patentes concedidas por país (1980-2002)

\begin{tabular}{lcccc}
\hline \multirow{2}{*}{ País } & \multicolumn{2}{c}{$\mathbf{1 9 8 0 - 1 9 9 0}$} & \multicolumn{2}{c}{ 1991-2002 } \\
\cline { 2 - 5 } & Cantidad & porcentaje & Cantidad & Porcentaje \\
\hline EUA & 223 & 24 & 1090 & 44,2 \\
Europa & 504 & 54,2 & 1087 & 44,1 \\
Japón & 175 & 18,8 & 197 & 8.0 \\
México & 17 & 1,8 & 27 & 1,1 \\
Otros & 11 & 1,2 & 63 & 2,5 \\
Total & 930 & 100 & 2464 & 100 \\
\hline
\end{tabular}

Fuente: Aboites y Soria, 2008. 
El IMPI proporciona información de las solicitudes de patentes y de las concedidas para los medicamentos. En una muestra de las marcas pioneras para antibióticos se encontró que la mayoría de ellas extendió su patente más de una ocasión. Durante el proceso de extensión de la patente las empresas aplicaron solicitudes y en algunos casos no se obtuvieron resultados favorables. Sin embargo, la innovación comercial forma parte de los atributos del medicamento pionero lo que permite diferenciarlo de sus rivales. De hecho, el 66\% de los casos analizados extendió su patente, y el 33\% restante no lo hizo en el período 1980-2010 "SIGA" (cuadro 6).

Cuadro 6. Extensiones de patente para 25 casos de antibióticos (1980-2010)

\begin{tabular}{lcccc}
\hline \multicolumn{1}{c}{ Empresa } & Marca & Extendió patente & Núm. de Solicitudes & Patentes \\
\hline (Glaxo) & Amoxil & Sí & 4 & 3 \\
Glaxo & Augmentin & Sí & 12 & 7 \\
Pfizer & Azitrocin & Sí & 11 & 9 \\
Eli- Lilly & Keflex & Sí & 6 & 5 \\
Eli-Lilly & Ceclor & No & 1 & 1 \\
Bristol-Myers Squibb & Pentrexil & Si & 7 & 6 \\
Glaxo-SmithKline & Zinnat & No & 1 & 1 \\
Bristol-Myers Squibb & Brispen & No & 2 & 2 \\
Bayer & Ciproxina & Sí & 3 & 2 \\
Abbott & Klaricid & Sí & 6 & 4 \\
Pharmacia Upjhon & Dalacin & Si & 3 & 2 \\
Eli-Lilly & Ilosone & Sí & 7 & 1 \\
Upjohn & Kaopetate & No & 1 & 10 \\
Pfizer & Acromicina & Sí & 14 & 1 \\
Roche & Bactrim & No & 1 & 5 \\
\hline Total & Sí extendieron & 10 & No extendieron & 34 \\
Total en & \% & 66 & & \\
\hline
\end{tabular}

Fuente: Elaboración propia con datos de IMPI SIGA, 2014.

\section{b. Barreras en la distribución mayorista}

El eslabón en la cadena de distribución mayorista es fundamental porque, como comentamos dos empresas compartían más de la mitad de las ventas en el mercado de distribución mayorista; por ende los medicamentos que se quieran distribuir en escala nacional o regional se enfrentarán a las condiciones o requisitos exigidos por las empresas de distribución mayorista los cuales son objeto de demanda en la Comisión Federal de Competencia.

La empresa que demandó a las dos grandes distribuidoras fue laboratorios "Roche", que argumentó ante la Comisión Federal de Competencia presuntas prácticas o condiciones que "Casa Saba" exigía a "Roche" para continuar distribuyéndole medicamentos, 
como la disminución de cuatro puntos porcentuales en el precio de toda la gama de medicamentos cuando la farmacéutica no aceptó dicha condición, la distribuidora dejó de inmediato de hacer pedidos. Según la empresa farmacéutica una semana después la distribuidora "Nacional de Drogas" exigió condiciones semejantes para poder continuar distribuyéndole productos y el resultado fue el mismo, porque la empresa negó sujetarse a las condiciones de las distribuidoras (Comisión Federal de Competencia, 2004).

Según el expediente DE-35-2004 de la Comisión Federal de Competencia, las distribuidoras exigieron la fijación de un precio de farmacia, es decir, uno máximo al que se pueda distribuir. Según "Roche" dicha práctica es un mecanismo de coordinación de oferta de precios entre las dos más grandes distribuidoras del país. Al existir antecedentes de prácticas monopólicas y dificultades para negociar la distribución mayorista de la industria, observamos que los medicamentos genéricos intercambiables son distribuidos en menor medida $(18,8 \%)$ que los que poseen marcas comerciales $(81,12 \%)$, sean pioneros o con marca de una muestra de antibióticos de patente expirada en 2012 (cuadro 7).

$\begin{gathered}\text { Cuadro } \\
\text { 7. Población de una muestra antibióticos distribuid } \\
\text { por "Nacional de Drogas" NADRO (mayo 2015) }\end{gathered}$
\begin{tabular}{lcc}
\hline Medicamento & Total & Total en \% \\
\hline Con Marca & 417 & 81,12 \\
G.I & 97 & 18,88 \\
Total & 514 & 100 \\
G.I & 97 & 100 \\
a) Extranjeros & 30 & 30,92 \\
b) Nacionales & 67 & 69,18 \\
\hline
\end{tabular}

Fuente: Elaboración propia con datos de "NADRO", Mayo (2015)

La situación anterior se debe a la pequeña estructura financiera que poseen las empresas de genéricos para enfrentar las condiciones impuestas por la distribuidora "Nacional de Drogas" para poder prestar sus servicios; por tanto buscan otras compañías de distribución con menor alcance y cobertura en el territorio nacional.

\section{c. Barreras en el sector detallista}

Anteriormente hemos señalado que las farmacias que pertenecen a las cadenas autoservicios y las de cadena han aumentado su participación de mercado en el sector mayorista. Las mencionadas en diversas ocasiones incurren en prácticas comerciales que como se vio pueden dificultar la presencia de los medicamentos genéricos a su exhibición.

Las cadenas de autoservicio rentan los espacios de sus anaqueles y dependiendo del lugar de ubicación existirá un costo así como un compromiso de abastecimiento de producto en la tienda (Mendoza, 2012). Según la autora, esas cadenas frecuentemente fijan 
requisitos a las empresas para poder colocar sus productos y exhibirlos en la tienda; si no se cubren, se rechaza la solicitud.

La consultora IMS Health (2013), indica que los laboratorios farmacéuticos están viendo en el segmento OTC una buena oportunidad para lanzar productos de marca, con márgenes de ganancia más altos que los productos genéricos y con un mayor volumen de venta. Como señala Mendoza esta estrategia permite a las empresas ubicar sus productos en los principales anaqueles de diversos puntos de venta en farmacia de prestigio. En el área de medicamentos libres de prescripción logran una mayor exhibición de los mismos y ubicar demostradoras o promotoras para explicar el uso de los productos así como de cosméticos, influyen en la decisión de compra de los consumidores.

\section{PATRONES DE COMPETENCIA Y SUS ASOCIACIONES EN LOS PRECIOS DE LOS ANTIBIÓTICOS DE PATENTE EXPIRADA}

Este subapartado dentro de la sección 2 tiene como objetivo presentar los resultados del modelo econométrico que analiza la asociación de las estrategias de competencia y los precios de las empresas de medicamentos de patente expirada. Se utilizaron varias fuentes de información. Una es el listado de precios de una de las distribuidoras líderes en el mercado farmacéutico, como es Nacional de Drogas o NADRO por sus siglas (World Pharmaceutical Markets, 2005). Complementamos la información con el Diccionario de especialidades farmacéuticas "PLM Vademecum", que reporta información de las empresas fabricantes, presentaciones de medicamentos y modo de empleo. Por último, se utilizó información de empresas procedentes de las cámaras y asociaciones empresariales, como la Cámara Nacional de la Industria Farmacéutica (Canifarma), Asociación Nacional de Fabricantes de Medicamentos (ANAFAM), y la Asociación Mexicana de Industrias de Investigación Farmacéutica (AMIIF).

La muestra se compone de $514^{1}$ antibióticos con patente expirada e indica que el 55,06\% de los productos farmacéuticos los comercializan empresas nacionales, y el $44,94 \%$ por compañías extranjeras, como se aprecia en el cuadro 7 . Con el fin de examinar las características de la muestra hemos elegido distinguir los productos según sean pioneros, seguidores con marca y G.I (Asociación Mexicana de Fabricantes Genéricos Intercambiables); las modalidades de diferenciación más frecuente; la marca; el número de presentaciones (tabletas, suspensión, y presentaciones de uso pediátrico); el tipo de dosificación (dosis única, dos, tres o más por día); estructura de mercado (nivel reducido de competidores y procedencia comercial) y la selección en el cuadro básico de la Secretaría de Salud (política pública). A continuación se presentan las estadísticas descriptivas a manera de antecedente.

\footnotetext{
1 Componentes químicos de la muestra como la amoxicilina simple y compuesta, ampicilina simple y con dicloxacilina, dicloxacilina simple, trimetropina con sulfatamexazol, claritrocimina, ciprofloxacino, eritromicina, azitromicina, levofloxacino, ofloxacino, cefaclor, cefadroxilo, cefalexina, loperamida, caolín-pectina y tetraciclina.
} 


\section{Hechos estilizados}

La información del cuadro 8 muestra que la contribución de las empresas nacionales a la innovación de medicamentos (pioneros) es mínima (5,81\% del total de medicamentos pioneros) y que ahí predominan las compañías extranjeras. En cambio, estas comercializan medicamentos genéricos con marca (63,34\% de la población de seguidores) y genéricos intercambiables $(69,39 \%$ de los G.I).

Cuadro 8. Características de la muestra por medicamento, origen de capital, línea de presentaciones y dosificación

\begin{tabular}{lcccccccccc}
\hline & Extranjero & Nacional & Total & Reducida & Amplia & Total & 1 dosis 2 dosis & Más de & Total \\
\hline Marca & & & Muestra & $\begin{array}{c}\text { Menor } \\
\text { a 5 }\end{array}$ & $\begin{array}{c}\text { Mayor } \\
\text { a 5 }\end{array}$ & Muestra & x día & x día & 2 dosis & Muestra \\
\hline Original & 81 & 5 & 86 & 22 & 64 & 86 & 20 & 30 & 36 & 86 \\
$\%$ & 94,19 & 5,81 & 100 & 25,58 & 74,42 & 100 & 23,26 & 34,88 & 41,86 & 100 \\
Seguidor & 120 & 210 & 330 & 246 & 84 & 330 & 41 & 128 & 161 & 330 \\
$\%$ & 36,36 & 63,64 & 100 & 74,55 & 25,45 & 100 & 12,42 & 38,79 & 48,79 & 100 \\
G.I & 30 & 68 & 98 & 87 & 11 & 98 & 12 & 33 & 53 & 98 \\
$\%$ & 30,61 & 69,39 & 100 & 88,78 & 11,22 & 100 & 12,24 & 33,67 & 54,08 & 100 \\
Total & 231 & 283 & 514 & 355 & 159 & 514 & 73 & 191 & 250 & 514 \\
$\%$ & 44,94 & 55,06 & 100 & 69,07 & 30,93 & 100 & 14,2 & 37,16 & 46,84 & 100 \\
\hline
\end{tabular}

Fuente: Elaboración propia con datos de la Distribuidora "Nacional de Drogas" o NADRO, mayo (2015) y Diccionario PLM Vademecum 2015

En relación con la marca y las dosificaciones observamos que los medicamentos originales o pioneros poseen líneas de presentaciones de más de cinco productos en sus respectivas marcas $(74,42 \%$ del total de productos pioneros), y el $58,14 \%$ de ellos se aplican cada 24 o 12 horas en un tratamiento. En cambio, los genéricos con marca (seguidores) tienen líneas de productos menores a cinco presentaciones ( $74,55 \%$ de la población de seguidores), y el 51,21\% de los citados están diseñados para administrarse cada 24 y 12 horas. Como es de esperarse, solo el $11 \%$ de los genéricos intercambiables tiene líneas de presentación amplias y el 46\% de ellos se administran cada 24 y 12 horas (cuadro 8).

La muestra indica que solo el $26,74 \%$ de los medicamentos pioneros forman parte del cuadro básico y el 22,09\% de ellos conforman escenarios de baja competencia (menos de cinco competidores). En segundo lugar, se ubican los genéricos con marca o productos seguidores donde el $41,52 \%$ del total de estos, pertenecen al cuadro básico y el 5,45\% integran nichos de mercados con poca competencia (cuadro 9).

En tercer lugar, el 51\% de los G.I. se ubican en el cuadro básico en mayor medida que los dos productos farmacéuticos mencionados, porque solo una minoría (3\% del total de G.I.), se ubican en nichos de mercado de baja competencia (cuadro 9). 
Cuadro 9. Medicamentos pioneros, genéricos con marca e intercambiables, y su relación con el cuadro básico y el nivel reducido de competidores

\begin{tabular}{lccccccccc}
\hline Medicamento & \multicolumn{3}{c}{ Cuadro Básico } & \multicolumn{3}{c}{$\begin{array}{c}\text { Nivel reducido } \\
\text { de competidores }\end{array}$} \\
\hline & Sí & No & Total & Menos de 5 & Más de 5 & Total & Importado & Nacional & Total \\
\hline Marca & & & & & & & & & \\
Pionero & 23 & 63 & 86 & 19 & 67 & 86 & 8 & 78 & 86 \\
$\%$ & 26,74 & 73,26 & 100 & 22,09 & 77,91 & 100 & 9,30 & 90,70 & 100 \\
Seguidor & 137 & 193 & 330 & 18 & 312 & 330 & 46 & 284 & 330 \\
$\%$ & 41,52 & 58,48 & 100 & 5,45 & 94,55 & 100 & 13,94 & 86,06 & 100 \\
G.I & 49 & 49 & 98 & 3 & 95 & 98 & 18 & 80 & 98 \\
$\%$ & 50 & 50 & 100 & 3,06 & 96,94 & 100 & 18,37 & 81,63 & 100 \\
Total & 209 & 305 & 514 & 40 & 474 & 514 & 73 & 441 & 514 \\
$\%$ & 40,66 & 59,34 & 100 & 7,78 & 92,22 & 100 & 14,20 & 85,80 & 100 \\
\hline
\end{tabular}

Fuente: Elaboración propia con datos de "Nacional de Drogas", marzo (2014) y mayo (2015) y del cuadro básico de medicamentos (2015).

En lo que respecta a la procedencia comercial (medicamentos de importación o de fabricación nacional), observamos que la cantidad de medicamentos importados es reducida en la muestra, porque el $85,80 \%$ de los fármacos se elaboran en México (cuadro 9), aunque los medicamentos genéricos con marca son el rubro donde más cantidad de productos importados existen (13,94\% del total de genéricos con marca).

Como información adicional que fundamenta la forma de dosificación y las líneas de presentaciones como medio de diferenciación de producto, las relacionamos con el cuadro básico para analizar el índice de beneficio en salud frente a un bajo costo desde la perspectiva fármaco-económica un medicamento debe ser efectivo a un costo accesible.

El cuadro 10 indica que el 63,2\% de los medicamentos con más de dos aplicaciones por día pertenecen a la política pública citada. En cambio, el $73 \%$ de los productos farmacéuticos de dosis única y el $87,43 \%$ de los que se aplican dos veces en un tratamiento, no pertenecen al cuadro básico lo que permite plantear que los productos de una y dos dosis por día en un tratamiento son innovaciones de productos antecesores que pertenecen al cuadro básico, aunque no necesariamente son más efectivos en términos terapéuticos.

Por otro lado, se puede apreciar la capacidad de diferenciación de producto porque los productos que cuentan con una línea de presentaciones amplias (más de 5), 64.15 por ciento de dichos casos no están apegados a la política pública es decir la innovación comercial no necesariamente es económica. Contrariamente los casos de productos con líneas de presentaciones reducidas $(57,18 \%)$ se asocian en mayor medida a la política pública (cuadro 10). 
Cuadro 10. Características de la muestra, cuadro básico, presentaciones y dosificaciones

\begin{tabular}{lccccccccc}
\hline Medicamento & \multicolumn{2}{c}{ Cuadro Básico } & \multicolumn{3}{c}{$\begin{array}{c}\text { Nivel reducido } \\
\text { de competidores }\end{array}$} \\
& Sí & No & Total & Menos de 5 & Más de 5 & Total & Importado & Nacional & Total \\
\hline Marca & & & & & & & & & \\
Pionero & 23 & 63 & 86 & 19 & 67 & 86 & 8 & 78 & 86 \\
$\%$ & 26,74 & 73,26 & 100 & 22,09 & 77,91 & 100 & 9,30 & 90,70 & 100 \\
Seguidor & 137 & 193 & 330 & 18 & 312 & 330 & 46 & 284 & 330 \\
$\%$ & 41,52 & 58,48 & 100 & 5,45 & 94,55 & 100 & 13,94 & 86,06 & 100 \\
G.I & 49 & 49 & 98 & 3 & 95 & 98 & 18 & 80 & 98 \\
$\%$ & 50 & 50 & 100 & 3,06 & 96,94 & 100 & 18,37 & 81,63 & 100 \\
Total & 209 & 305 & 514 & 40 & 474 & 514 & 73 & 441 & 514 \\
$\%$ & 40,66 & 59,34 & 100 & 7,78 & 92,22 & 100 & 14,20 & 85,80 & 100 \\
\hline
\end{tabular}

Fuente: Elaboración propia con datos de "Nacional de Drogas", marzo (2014) y mayo (2014), PLM “Vademecum” y el cuadro básico de medicamentos (2015).

Las variables de juego en un esquema Stackelberg y de diferenciación por marca son los productos originales (marcas pioneras) que tienen expiración de patente y marcas seguidoras (genéricos con marca) que como se mencionó registra todos los productos de competidores que se comercializan bajo una marca y no por su denominación genérica, la de Comercio Internacional (procedencia, importado-nacional), estructura de mercado (número reducido de competidores), política pública (cuadro básico) y las que engloban las características y diferenciación de producto son (presentaciones, dosificaciones, espectraje). En la diferenciación de producto, las innovaciones comerciales e intentos de extensión de patente de la marca pionera se traducen en un aumento en la cantidad de presentaciones y en las dosificaciones de un tratamiento, tal y como se revisó en el capítulo dos en el apartado de diferenciación y barreras a la entrada. Sin embargo, en otros casos no se mejora por la dosificación, si no en el modo de empleo (supositorios, pomadas, inyecciones, etc.), es así que esta acción solo puede verse reflejada en la cantidad de presentaciones de una marca.

Por otro lado, el nivel de espectraje es un elemento a considerar porque los medicamentos que tratan enfermedades de diversas áreas terapéuticas (diarreas, males respiratorios, infecciones de la vista, de tejido etc.), pudieran ser más caros que los homólogos específicos (medicamentos que atienden una enfermedad), debido a los múltiples beneficios que ofrecen al paciente. 
Cuadro 11. Características de las variables a analizar en el modelo econométrico

\begin{tabular}{ll}
\hline \multicolumn{1}{c}{ Variable (Clasificación) } & \multicolumn{1}{c}{ Composición } \\
\hline Precio (Numérica) & $\begin{array}{l}\text { Calculada a precio por dosis para equiparar las diferentes unidades de } \\
\text { medida y se transformó a logaritmo natural }\end{array}$ \\
Procedencia (dummy) & Importado (1) Nacional (0) \\
Competidores & Numérica \\
Presentaciones (dummy) & (1) líneas mayores a 5 presentaciones (0) menores a 5 presentaciones \\
Dosificación única (dummy) & Toma única (1) No es de toma única (0) \\
Dos dosis por día (dummy) & Más de dos tomas (1) No se administran más de dos tomas (0) \\
Cuadro básico (dummy) & Pertenece (1) No pertenece (0) \\
Pionero (dummy) & Pionero (1) No es pionero (0) \\
Seguidor (dummy) & Es seguidor (1) No es seguidor (0) \\
Amplio espectro (dummy) & Es amplio (1) No es amplio (0) \\
\hline
\end{tabular}

Fuente: Elaboración propia.

El cuadro 11 ilustra las variables del modelo econométrico de corte transversal para el período 2014 y 2015. Diversos trabajos utilizan trabajos econométricos para analizar la paradoja de los genéricos. La mayoría de ellos son estudios longitudinales, como las regresiones panel y series de tiempo con variables transformadas a logaritmos (Frank y Salkever; 1997, Saha et al., 2006; Reagan, 2008; Vandoros y Kanavos, 2013; Mueller y Frenzel, 2015). Otros realizan estadística multivariada para analizar el comportamiento de precios (Grabowsky y Vernon, 1992). Sin embargo, este artículo propone realizar un modelo de regresión transversal porque se busca establecer asociaciones entre diferenciación de producto y precios. Asimismo, pretende evaluar los factores asociados a incrementos de precios. Contrario a los trabajos propuestos porque los mencionados buscan evaluar la permanencia de precios ante la entrada de competencia de las marcas pioneras y el comportamiento de precios de los distintos competidores de genéricos, utilizando una variable de cambio estructural para medir la paradoja. Este pretende establecer asociaciones después de la expiración razón por la cual no es necesario realizar una regresión panel y que no es fácil obtener información de precios de las distribuidoras de medicamentos, es decir se intenta aportar información que explique la paradoja de los genéricos y no fundamentar la presencia de la misma puesto que ya hay evidencia de las misma en el mercado mexicano (De la Garza, 2014 y Cofece, 2017). 


\section{ESTIMACIONES DE LOS PATRONES DE COMPETENCIA Y SUS ASOCIACIONES A LAS VARIACIONES DE PRECIOS}

Esta sección comienza con el análisis de las variables y sus asociaciones porcentuales sobre el precio por dosis de los medicamentos. Por tanto, se elabora la especificación del modelo para tratar la regresión lineal con variables numéricas, dummy o binarias y su efecto en una variable logarítmica (precio). La forma en se determinan las asociaciones log-dummy que determinan los crecimientos porcentuales, viene en la sección de Anexo.

La especificación de los modelos de corte transversal es la siguiente:

$$
\begin{aligned}
& L_{n} \text { precio }=\beta 0+\beta 1 \text { original }+\beta 2 \text { seguidor }+\beta 3 \text { procedencia } \\
& \quad+\beta 4 \text { presentaciones grandes }+\beta 5 \text { competidores } \\
& \quad+\beta 6 \text { cuadro básico }+\beta 7 \text { dosis única }+\beta 8 \text { dos dosis por día } \\
& \quad+\beta 9 \text { amplio espectro }
\end{aligned}
$$

El cuadro 12 muestra los resultados de las regresiones econométricas. Puede verse que ambos modelos poseen un coeficiente de determinación de 0,598 para ambos períodos, es decir, el 59,8\% de la variabilidad de los precios se explica por las variables que componen los modelos. Asimismo, se reporta que todas las variables explicativas fueron significativas al $5 \%$, salvo la variable de competencia y el espectraje ${ }^{2}$ que puede tener un medicamento de patente expirada.

Las tres pruebas realizadas para analizar el carácter robusto de los resultados fueron satisfactorias y pueden apreciarse en la sección de Anexos. A continuación presentamos los resultados de los modelos para los períodos 2014 y 2015. Comenzamos con la diferenciación de producto.

\section{a. Diferenciación de producto}

Los resultados permiten observar que los medicamentos dosificación única por día (tomada cada 24 horas) tuvieron un incremento de 649 y 647 por ciento sobre los medicamentos que no cumplen la condición mencionada (antibióticos que se administran dos, tres y cuatros veces por día en un tratamiento), para los períodos 2014 y 2015 respectivamente (cuadro 12 ).

Por su parte, los medicamentos de dos aplicaciones por día en un tratamiento tienen variaciones de precios menores que los de aplicación única. Aunque, no es despreciable porque dichos productos obtuvieron un crecimiento de 125 y 121 por ciento en sus precios que los medicamentos de tres o más aplicaciones en 2014 y 2015 respectivamente.

\footnotetext{
2 Amplio espectro. Significa que un medicamento puede combatir varias enfermedades de distintas clases subclases terapéuticas.
} 
Los resultados coinciden con los hallazgos encontrados en Reino Unido (Kanavos, Costa-Font y Seeley, 2008) donde la correlación de las estrategias de diferenciación fueron positivos. Sin embargo, el estudio difiere un poco de nuestros resultados porque no desdeñaron detalladamente la variable de diferenciación de producto, ya que su variable dummy conjuntaba los cambios en presentaciones, componentes químicos y dosificaciones en una sola variable (cuadro 12).

Cuadro 12. Resultados de la Estimación econométrica

\begin{tabular}{lcccccc}
\hline \multirow{2}{*}{ Variable } & \multicolumn{3}{c}{ Modelo para 2014 } & \multicolumn{3}{c}{ Modelo para 2015 } \\
\cline { 2 - 7 } & Coeficiente & $\mathrm{p}>\mathrm{t}$ & Efecto marginal\% & Coeficiente & $\mathrm{p}>\mathrm{t}$ & Efecto marginal $\%$ \\
\hline Competidores & $-0,1602186$ & 0,151 & & $-0,1594256$ & 0,153 & \\
Pionero & 0,7305655 & 0,000 & 107,63 & 0,8185054 & 0,000 & $126-71$ \\
Seguidor & 0,5149614 & 0,000 & 67,36 & 0,5389853 & 0,000 & 71,43 \\
Procedencia & 0,2110035 & 0,010 & 23,49 & 0,235514 & 0,006 & 26,56 \\
Amplio espectro & $-0,0178909$ & 0,790 & & $-0,0129805$ & 0,849 & \\
Pres. grandes & 0,1707424 & 0,020 & 18,62 & 0,1783701 & 0,014 & 19,53 \\
Dosis única & 2,014002 & 0,000 & 649,32 & 2,012098 & 0,000 & 647,90 \\
2dosis x dia & 0,8127327 & 0,000 & 125,41 & 0,7932073 & 0,000 & $121-05$ \\
Cuadro básico & $-0,1567197$ & 0,030 & $-14,51$ & $-0,1687714$ & 0,017 & $-15,53$ \\
Obs. & 514 & $\mathrm{R} 2$ & 0,5984 & 514 & $\mathrm{R} 2$ & 0,5984 \\
\hline
\end{tabular}

Fuente: Elaboración propia.

En lo que concierne a la cantidad de presentaciones que puede tener una marca, observamos incrementos de 18,4 y 19,3 por ciento en sus precios, respecto a las marcas que no pertenecen a dicha categoría para los años mencionados. Los resultados mencionados, confirman los hallazgos de Hong et al. (2005), quienes encuentran asociaciones positivas en los precios de los medicamentos en casos donde existió extensión de patente, frente a los que carecen de esta.

\section{a. Diferenciación de marca}

Las estimaciones para las marcas mencionadas, indican que ser el primero tiene ventajas en la fijación de precios porque les permite tener incrementos de 107\% y 126\% (cuadro 13) sobre los productos farmacéuticos que no contienen dichas características (competidores de genéricos con marca y sin marca). Los resultados son consistentes con los obtenidos por Grabowsky y Vernon (1992), Frank y Salkever, (1997) y Reagan (2008). Para el caso de México, los resultados son confirmados con los reportados de la COFECE (2017) quienes encuentran asociaciones positivas del medicamento pionero. 
Bajo un contexto similar de diferenciación por una marca reconocida, hay medicamentos genéricos que optarán por seguir el patrón de competencia de la marca pionera, es decir introducir productos con diferenciaciones similares a la marca líder y con un nombre comercial respaldado por un laboratorio de prestigio, por ende hay posibilidad de fijar precios elevados. Lo anterior se traduce variaciones positivas en el mercado de patente expirada, aunque en menor medida porque los medicamentos genéricos con marca tuvieron un incremento de 67,10 y 71,10 por ciento en el precio, respecto a las versiones de genéricos sin marca (cuadro 13). Los resultados reafirman los hallazgos de Grabowsky y Vernon (1992), quienes encuentran que los precios de los medicamentos genéricos con marca son mayores que los de sus homólogos sin marca, y coincide con las correlaciones descritas por Reiffen y Ward (2007) porque encuentran una asociación negativa menor sobre los precios al ingresar un medicamento genérico diferenciado, que en mercados donde deciden no incursionar, es decir inciden positivamente en los precios.

Por otro lado, confirmamos los resultados en Hollis (2005), porque existen medicamentos genéricos con marca que son fabricados las empresas pioneras y tienen capacidad de fijar precios mayores que sus rivales en el mercado de genéricos. Una explicación a la diferenciación según el autor, es la segmentación de mercados porque las empresas buscarán ofrecer medicamentos con marca en un segmento donde hay lealtad a la marca. Finalmente no fue significativo el nivel de espectraje, es decir que un medicamento sea multifuncional para tratar distintos padecimientos infecciosos no necesariamente estará asociado a incrementos en precios.

\section{b. Estructura de mercado}

El nivel o grado competencia no tiene asociación alguna sobre el precio de los medicamentos, según la significancia del modelo. Los hallazgos debaten y contraponen los planteamientos sobre las asociaciones negativas que puede tener el grado de competencia en los precios, como los obtenidos en Saha et al. (2006), quienes encuentran una asociación negativa entre el precio de los medicamentos originales y la cantidad de incumbentes. En este sentido, contradicen los hallazgos de Reiffen y Ward (2005), quienes predicen el precio previo a la expiración de patente, que podrían tener los medicamentos genéricos conforme aumenta o disminuye la cantidad de competidores y los hallazgos de Caves et al. (1991), quien plantea descensos en los precios por rangos acorde a la cantidad de competidores.

\section{c. Comercio internacional}

Los resultados obtenidos muestran que los antibióticos importados tienen incrementos de 23,49 y 26,5 por ciento sobre el precio fijado de los medicamentos de fabricación nacional (cuadro 13). Es decir, los medicamentos importados no necesariamente serán 
más económicos que los de fabricación nacional, en contraposición a los supuestos teóricos y clásicos del comercio internacional que aluden a la importación como ventaja comparativa para las naciones en diversidad de productos y mejores precios. Es importante mencionar que dicha variable no tiene antecedentes en la literatura revisada. Aunque respaldan planteamientos sobre el comercio intraindustrial en la industria, es decir hay empresas que importan medicamentos genéricos similares a los de fabricación nacional desde la apertura de la apertura comercial en México y esta práctica ha mostrado un crecimiento notable porque no todos los medicamentos que entran al país necesariamente es por falta de ellos; si no por diferenciación de producto (Ríos, 2018).

\section{d. Política pública}

El cuadro básico de medicamentos comprende los medicamentos que son efectivos en términos de costo-beneficio en el mercado institucional o sector salud público. No obstante, la política pública tiene asociaciones negativas en el mercado farmacéutico privado. Los resultados estimados muestran que los componentes químicos y presentaciones que se apegan al cuadro básico presentaron decrementos de $-14,75 \mathrm{y}$ $-15,8$ por ciento en sus precios para los períodos 2014 y 2015 (cuadro 13), respecto a las versiones que no pertenecen a un esquema de costo-beneficio. Los resultados son contradictorios a los obtenidos por analistas como De la Garza (2014) quien encuentra asociaciones positivas en los precios de los medicamentos al insertarse al cuadro básico de medicamento.

Los hallazgos nos permite deducir que la política pública cumple con una función social en el precio de los medicamentos en ciertos componentes y presentaciones para el mercado farmacéutico privado, es decir, la política pública tiene alcances en ambos segmentos de mercado.

Finalmente apreciamos que los precios no presentan cambios bruscos de 2014 a 2015, es decir, no hay grandes diferencias entre los períodos de estudio y el nivel de significancia en las regresiones lo que permite validar consistencia en las variables son consistentes del modelo econométrico. Asimismo, reportamos que las asociaciones en los precios aumentaron de 2014 a 2015 en la marca pionera, en la seguidora, en la cantidad de presentaciones y en la procedencia comercial de los medicamentos y en la dosificación única. Al contrario, la variable de dos dosificaciones presentó un leve descenso de un período a otro, y la política pública aumentó su descenso de 2014 a 2015, lo que implica una efectividad de esta en los antibióticos ofertados para el mercado nacional. 


\section{CONCLUSIONES}

El presente artículo demuestra que la competencia en el mercado de patente expirada no es perfecta, con la presencia de gran heterogeneidad de precios. Los resultados indican que la variación de precios es explicada por las mejoras de un producto mediante mejoras en las presentaciones (dosificaciones y nuevos modos de empleo) y posicionamiento competitivo en una marca (pionera y seguidoras), es de suma importancia reflexionar respecto a las ventajas que tiene una empresa establecida. Sin dejar de lado, que la procedencia comercial es otro elemento explicativo al fenómeno, donde se puede apreciar que en cierta medida el comercio en el mercado de genéricos no necesariamente obedece al principio de la complementariedad expuesto por la ventaja absoluta y comparativa. Más bien, obedece a planteamientos de la nueva teoría del comercio internacional, específicamente a la presencia de diferenciación de producto y comercio intraindustrial. Lo anterior son los factores que explican la paradoja de los genéricos donde la segmentación de mercados es vital para la ejecución o permanencia de la misma y que la empresa dominante tiene capacidad para preservar la mitad del mercado. A este fenómeno, se le ha buscado contrarrestar con políticas públicas orientadas a la regulación de precios evitar las asimetrías que hay en las participaciones de mercado en volumen y en valores, tanto de las marcas pioneras, como las de las seguidoras En el caso de México, se encuentra que el cuadro básico de medicamentos, que es una política capaz de reducir las dispersiones entre los medicamentos de patente expirada. Sin embargo es limitada porque no todas las presentaciones y antibióticos en el mercado lo conforman, lo que deja una línea abierta a la evaluación de la misma y más ahora que el nuevo gobierno entrante de López Obrador pretende desaparecerla, lo que cambiará en un futuro próximo un nuevo fenómeno económico en materia de precios. Finalmente, se aprecia que los medicamentos genéricos que compiten por precios (genéricos sin marca) pudieran tener mayor penetración, si se prestara atención a las diversas barreras que enfrentan en los diversos eslabones de la cadena de valor, así como, en el ámbito legal donde se desenvuelven al expirar la patente. Es importante que el consumidor tenga mayor conocimiento para la adquisición de medicamentos a precios económicos. La experiencia ha europea ha demostrado que el precio de referencia pudiera ser una alternativa para buscar mayor penetración de genéricos a precios módicos y con la posibilidad de contener el gastos farmacéutico para los casos donde hay cobertura universal de atención y financiamiento de medicamentos a los usuarios. Por otro lado, donde hay casos de sistemas de salud bipartitas (público y privado), la política ha demostrado ser eficiente (Ríos, 2015). Sin embargo, esto depende también de las formas en que se organiza la industria en cada país y las políticas encaminadas a promover medicamentos accesibles para la población. 


\section{ANEXO}

\section{Pruebas estadisticas de los modelos econométricos}

La prueba Breusch-Pagan nos muestra que la varianza de los errores es homogénea para ambos períodos

\begin{tabular}{ll}
\hline \multicolumn{1}{c}{ Breusch-Pagan (2014) } & \multicolumn{1}{c}{ Breusch-Pagan (201) } \\
\hline Weisberg test for heteroskedasticity & Weisberg test for heteroskedasticity \\
Ho: Constant variance & Ho: Constant variance \\
Variables: fitted values of lprecio & Variables: fitted values of lprecio \\
chi2 $(1)=3.17$ & chi2 $(1)=3.51$ \\
Prob $>$ chi $2=0.0750$ & Prob $>$ chi $2=0.0610$ \\
\hline
\end{tabular}

\section{Prueba Co-linealidad}

\begin{tabular}{lcclcc}
\hline & Vif (2014) & & \multicolumn{3}{c}{ Vif (2015) } \\
\hline Variable & VIF & 1/VIF & Variable & VIF & $1 /$ VIF \\
Original & 1,98 & 0,50409 & Original & 1,98 & 0,50409 \\
Seguidor & 1,6 & 0,626272 & Seguidor & 1,6 & 0,626272 \\
Dosisxdia & 1,46 & 0,686121 & Dosisxdia & 1,46 & 0,686121 \\
cuadbasico & 1,42 & 0,702314 & cuadbasico & 1,42 & 0,702314 \\
Presgde & 1,32 & 0,757564 & Presgde & 1,32 & 0,757564 \\
dosisunica & 1,22 & 0,816486 & dosisunica & 1,22 & 0,816486 \\
amplioespectro & 1,16 & 0,861113 & amplioespectro & 1,16 & 0,861113 \\
pocoscomp & 1,15 & 0,87304 & Pocoscomp & 1,15 & 0,87304 \\
procedencia & 1,05 & 0,951893 & Procedencia & 1,05 & 0,951893 \\
Mean VIF & 1,37 & & Mean VIF & 1,37 & \\
\hline
\end{tabular}

La prueba indica que no hay colinealidad entre los regresores, puesto que la media es menor a 7 . 


\section{Test de Especificación}

La prueba indica que no existen errores de especificación con la prueba de Ramsey

ovtest (2014)

Ramsey RESET test using powers of the fitted values of lprecio

Ho: model has no omitted variables

$\mathrm{F}(3,501)=2.02$

Prob $>\mathrm{F}=0,1098$

ovtest (2015)

Ramsey RESET test using powers of the fitted of lprecio

Ho: model has no omitted variables

$\mathrm{F}(3,501)=1,94$

Prob $>\mathrm{F}=0,1227$

Según Greene (2012), un modelo con variable dependiente logarítmica y regresores dummy, se representa así:

$$
\ln Y=\beta 1+\beta 2 x+\beta 3 d+\varepsilon
$$

El coeficiente de la variable dummy, $d$ indica un cambio multiplicativo de la función. El porcentaje de cambio en $E[y \mid x, d=1]$ asociado a un cambio en $d$ es:

$$
\begin{aligned}
\%\left(\frac{\Delta E[y \mid x, d]}{\Delta d}\right) & =100 \%\left\{\frac{E[y \mid x, d=1]-E[y \mid x, d=0]}{E[y \mid x, d=0]}\right\} \\
= & 100 \%\left\{\frac{\exp (\beta 1+\beta 2 x+\beta 3 E[\exp (\varepsilon)]-\exp (\beta 1+\beta 2 x) E[\exp (\varepsilon)}{\exp (\beta 1+\beta 2 x) E[\exp (\varepsilon)]}\right\} \\
= & 100 \%[\exp (\beta 3)-1]
\end{aligned}
$$




\section{REFERENCIAS BIBLIOGRÁFICAS}

Aboites, Jaime y Manuel Soria (2008). Economía del conocimiento y propiedad intelectual: Lecciones para la economía mexicana. Ciudad de México: Siglo Veintiuno Editores-UAM Iztapalapa.

Bain, Joe (1956). Barriers to new competition. Cambridge: Harvard University Press. https://doi. org/10.4159/harvard.9780674188037

Barraza, Mariana y José Guajardo (2013). Descripción del sector farmacéutico en México, 2012. Recuperado de http://funsalud.org.mx/portal/wpcontent/uploads/2013/08/ DescripcionSF2012_Funsalud_vF-1401141.pdf (30 octubre 2018)

Bhat, Vasanthakumar (2005). Patent term extension strategies in the pharmaceutical industry. Policy and Law, 6(1), 109-122.

Caves, Richard, Michael Whinston y Mark Hurwitz (1991). Patent Expiration, Entry and Competition in the US Pharmaceutical Industry. Microeconomics 1980. pp. 1-65. Brooking Papers on Economic Activity. https://doi.org/10.2307/2534790

Clift, Charles (2008). The value of patent term extensions to the pharmaceutical industry in the USA. Journal of Generic Medicines, 5(3), 201-208. https://doi.org/10.1057/jgm.2008.6

COFECE (2004). Expediente No. DE-35-2004. En http://www.cofece.mx/docs/pdf/ de0352004resolucionvp.pdf (5 junio 2018).

COFECE (2017). Estudio en materia de libre concurrencia y competencia sobre los mercados de medicamentos con patentes vencidas en México. Recuperado de http://cofece. socialand-labs.com/tema-original/wp-content/uploads/2017/11/estudio-de-medicamentos_vf-baja-1.pdf (Abril 2018).

Danzon, Patricia y Michael Furukawa (2003). Prices and Availability of Pharmaceuticals: Evidence from Nine Countries. Health Affairs, 39(2), 521-536. https://doi.org/10.1377/ hlthaff.W3.521

Danzon, Patricia y Michael Furukawa (2008). International Prices and Availability of Pharmaceuticals in 2005. Health Affairs, 27(1), 221-233. https://doi.org/10.1377/ hlthaff.27.1.221

Danzon, Patricia y Michael Furukawa (2011). Cross-national Evidence on Generic Pharmaceuticals: Pharmacy vs. Physician-driven Markets. NBER Working Paper 17226. https://doi. org/10.3386/w17226

De la Garza Garza, Oscar Javier (2014). Tres ensayos sobre competencia económica. Tesis de doctorado en Ciencias Económicas. Facultad de Economía, Universidad Autónoma de Nuevo León. Recuperado de https://cd.dgb.uanl.mx/handle/201504211/16734

El Universal (2008). Se cotiza alto el precio de los anaqueles en autoservicios. Recuperado de http://archivo.eluniversal.com.mx/notas/528109.html (5 junio 2018)

Ferrara, Ida y Ying Kong (2008). Can health insurance coverage explain the generic competition paradox? Economic Letters, 101(1), 48-52. https://doi.org/10.1016/j.econlet.2008.04.011

Ferrándiz, Jorge (1999). The impact of generic goods in the pharmaceutical industry. Health Economics, 8(7), 599-612. https://doi.org/10.1002/(SICI)1099-1050(199911)8:7<599: :AID-HEC479>3.0.CO;2-K

Frank, Richard y David Salkever (1992). Pricing, Patent Loss and the Market For Pharmaceuticals. Southern Economic Journal, 2(59), 165-179. https://doi.org/10.2307/1060523

Frank, Richard y David Salkever (1997). Generic Entry and the Pricing of Pharmaceuticals. Journal of Economics \& Management Strategy, 6(1), 75-90. https://doi.org/ $10.1162 / 105864097567039$ 
Grabowsky, Henry y John Vernon (1992). Brand Loyalty, Entry, and Price Competition in Pharmaceuticals after the 1984 Drug act. Journal of Law and Economics, 32(2), 331-350. https://doi.org/10.1086/467257

Granier, Laurent y Sébastien Trinquiard (2010). Entry deterrence and mergers under price competition in pharmaceutical markets. Applied Economics, 3(42), 297-309. https://doi. org/10.1080/00036840701604495

Granier, Laurent y Sébastien Trinquiard (2012). Predation in off-patent drug markets. Applied Economics, 17(44), 2171-2186. https://doi.org/10.1080/00036846.2011.562169

Greene, William (2012). Econometric Analysis. New York: Pearson.

Guzmán, Alenka y María Pluvia (2004). Patentes en la industria farmacéutica de México: Los efectos en la investigación, el desarrollo y en la innovación. Comercio Exterior, 54(12), 1104-1121.

Hollis, Aidan (2002). The importance of being first: evidence from Canadian generic Pharmaceuticals. Health Economics, 11(8), 723-734. https://doi.org/10.1002/hec.698

Hollis, Aidan (2005). How do brands own generics affect generic prices? Review of Industrial Organization, 27(4), 329-350. https://doi.org/10.1007/s11151-005-5469-5

Hong, Soon, Marvin Shepherd, David Scoones y Thomas Wan (2005). Product-Line Extensions and Pricing Strategies of Brand-Name Drugs Facing Patent Expiration. Journal of Managed Care Pharmacy, 11(9), 746-754. https://doi.org/10.18553/jmcp.2005.11.9.746

Hurwitz, Michael y Richard Caves (1988). Persuasion or Information? Promotion and the Shares of Brand Name and Generic Pharmaceuticals. Journal of Law and Economics, 31(2), 299-320. https://doi.org/10.1086/467158

Iizuka, Toshiaki (2009). Generic Entry in a Regulated Pharmaceutical Market. Japanese Economic Review, 60(1), 63-81. https://doi.org/10.1111/j.1468-5876.2008.00465.x

Kamien, Morton, Israel Zang (1999). Virtual patent extension by cannibalization. South Economics Journal, 1(66), 117-131. https://doi.org/10.2307/1060838

Kanavos, Panos, Joan Costa-Font, Elizabeth Seeley (2007). Competition in Off-Patent Drug Markets: Issues, Regulation and Evidence. Working Paper. Recuperado de https://pdfs.semanticscholar.org/b590/b88ed48b51a07aa4c7a9ec1bc2992fa4d666.pdf (febrero 2018).

Kanavos, Panos, Joan Costa-Font, Elizabeth Seel (2008). Competition in Off-Patent Drug Markets: Issues, Regulation and Evidenc. Economic Policy, 23(55), 500-544. https://doi. org/10.1111/j.1468-0327.2008.00207.x

Kim, Linzu (1997). Imitation to Innovation: The Dynamics of Korea's Technological Learning. Boston: Harvard Business School Press.

Knobloch (2008). Mercado farmacéutico privado mexicano. Recuperado de http://www.pharmalink.ch/pdf/mfpm.pdf (6 de mayo 2016).

Knobloch (2009). Valor de la producción de los genéricos intercambiables de 2007 a 2009. En R. Maciel, Genéricos intercambiables y renovación de registros 2010. (AMEGI) en http:// www.dgplades.salud.gob.mx (6 de abril 2015).

Kong, Ying (2004). The Premium Price of generic to Brand names and pharmaceutical Price index. Applied Economics, 36(7), 731-737. https://doi.org/10.1080/0003684042000222106

Kong, Ying y James R. Seldon (2004). Pseudo-Generic Products and Barriers to Entry in Pharmaceutical Markets. Review of Industrial Organization, 25(1), 71-86. https://doi. org/10.1023/B:REIO.0000040524.32034.04 
Magazzini, Laura, Fabio Pammolli y Massimo Riccaboni (2004). Dynamic competition in pharmaceuticals. European Journal of Health Economics, 5(2), 175-182. https://doi. org/10.1007/s10198-003-0218-x

Mizrahi, Elías (2010). Regulación y competencia en el mercado de medicamentos: experiencias relevantes para América Latina. CEPAL Serie de Estudios y perspectivas, 121(2), 1-65.

MOÏSE, Pierre y Elizabeth DOCTEUR (2007). Pharmaceutical Pricing and Reimbursement Policies in Mexico OECD. Health Working Papers 25. Recuperado de http://apps.who. int/medicinedocs/en/d/Js19839en/

Mueller, Michael y Alexander Frenzel (2015). Competitive pricing within pharmaceutical classes: evidence on "follow-on" drugs in Germany 1993-2008. The European Journal of Health Economics, 1(16), 73-82. https://doi.org/10.1007/s10198-013-0555-3

Papanastasiou, Anastasios (2016). A Unifying Theory of the Generic Competition Paradox: Dynamic limit pricing with advertisement. Tesis de doctorado. Universidad de Guelph, Ontario Canadá. Recuperado de https://pdfs.semanticscholar.org/6250/973d38e228a4535ae8a5 a7c28988b0459c95.pdf

Pearce, John (2006). How companies can preserve market dominance after patent expire. Long Range Planning, 39(1), 71-87. https://doi.org/10.1016/j.lrp.2005.04.006

PM FARMA (2013). Proliferan fármacos de venta sin receta. Recuperado de http://www.pmfarma. com.mx/noticias/8747-proliferan-farmacos-de-venta-sin-receta.html (junio 2016).

Reagan, Tracy (2008). Generic entry, price competition, and market segmentation in the prescription drug market. International Journal of Industrial Organization, 26(4), 930-948. https://doi.org/10.1016/j.ijindorg.2007.08.004

Reiffen, David y Michael Ward (2007). Branded Generics as a strategy to limit cannibalization of Pharmaceutical markets. Managerial and Decision Economics, 28(4), 251-265. https:// doi.org/10.1002/mde.1339

Ríos, Eric (2015). Políticas públicas para regular el comportamiento del mercado de patente expirada. En Lilia Domínguez y Flor Brown (eds.), Los retos de la nueva política industrial (pp. 229-246). Ciudad de México: Universidad Nacional Autónoma de México, Facultad de Economía.

Ríos, Eric (2018). Comercio Intra-industrial en la industria farmacéutica mexicana en un contexto de apertura comercial (1993-2016). En Isaí Contreras, Patricia López y Eric Ríos (eds.), Los paradigmas en las dinámicas legales, económicas y de competitividad en el comercio internacional (pp. 83-106). Tolcayuca, Hidalgo: Ediciones de la Noche, Universidad Politécnica Metropolitana de Hidalgo.

Rodrigues, Vasco, Ricardo Gonçalves, Hélder Vasconcelos (2014). Anti-Competitive Impact of Pseudo-Generics. Journal of Industrial Competition and Trade, 14(1), 83-98. https://doi. org/10.1007/s10842-013-0154-0

Salud y Fármacos (2013). El mercado de genéricos en América Latina, y concretamente en México, está en franca expansión. Recuperado de http://www.saludyfarmacos.org/lang/ en/boletinfarmacos/boletines/ago2014/ago2014596/.

Saha Atanu, Henry Grabowsky, Howard Birnbaum, Paul Greenberg, Oded Bizan (2006). Generic Competition in the US Pharmaceutical Market. International Journal of the Economic Business, 13(1), 15-38. https://doi.org/10.1080/13571510500519905

Scherer, Mike (1993). Pricing, Profits, and Technological Progress in the Pharmaceutical Industry. Journal of Economic Perspectives, 7(3), 97-115. https://doi.org/10.1257/jep.7.3.97 
Schettino, Macario (2003). Introducción a la economía para no economistas. Ciudad de México: Pearson.

Scott, Fiona (2000). Barriers to entry, brand advertising, and generic entry in the US pharmaceutical industry. International Journal of Industrial Organization, 18(7), 1085-1104. https:// doi.org/10.1016/S0167-7187(98)00057-5

Scott, Fionna (1999). Entry decisions in the generic pharmaceutical industry. The RAND Journal of Economics, 30(3), 421-440. https://doi.org/10.2307/2556056

SIGA (2014). En http://siga.impi.gob.mx/content/common/principal.jsf (2 marzo 2016).

Stackelberg, Heinrich von (1952). The Theory of the Market Economy. Londres: Oxford University Press.

Suh, Dong-Churl, Willard Manning, Stephen Schondelmeyer y Ronald Hadsall (2000). Effect of multiple-source entry on price competition after patent expiration in the pharmaceutical industry. Health Services Research, 35(2), 529-547.

Tirole, Jean (1988). The Theory of Industrial Organzation. Massachussuets: MIT Press.

Vandoros, Sotiris y Panos Kanavos (2013). The generics paradox revisited: empirical evidence from regulated markets. Applied Economics Journal, 22(45), 3230-3239. https://doi.org/ 10.1080/00036846.2012.703313

Wiggins, Steven y Robert Maness (2004). Price competition in pharmaceuticals: the case of anti-infectives. Economic Inquiry, 42(2), 247-263. https://doi.org/10.1093/ei/cbh058

Documento recibido el 17 de diciembre de 2018

y aprobado el 26 de febrero de 2019 